\title{
Repercussões emocionais em pacientes em seguimento oncológico: ansiedade, depressão e qualidade de vida
}

\author{
Emotional repercussions in cancer patients: anxiety, depression and quality of life
}
Repercusiones emocionales en pacientes oncológicos: ansiedad, depresión y calidad de vida

Alyne Barbosa da Silva ${ }^{1 *}$, Heverton Valentim Colaço da Silva², Érika Neves de Barros².

\section{RESUMO}

Objetivo: Estimar a prevalência de sinais e sintomas de ansiedade e depressão e avaliar a qualidade de vida de pacientes em seguimento oncológico. Métodos: O respectivo estudo trata-se de um corte transversal, incluindo 246 pacientes que estão em seguimento oncológico. Utilizou-se questionário com variáveis sociodemográficas e informações clínicas, acrescido dos instrumentos: Escala Hospitalar de Depressão e Ansiedade (HADS) e World Health Organization Quality of Life (WHOQOL-bref). Resultados: A prevalência de ansiedade moderada a severa foi de 33,7\% (IC 95\%: 27,8 - 39,7) e de 13,4\% (IC 95\%: 9,1 - 17,7) para depressão moderada a severa. A qualidade de vida teve escore médio de 68,52 pontos. Os pacientes, que apresentaram sintomas de ansiedade e depressão moderada a severa, tiveram uma pior avaliação da qualidade de vida em todos os domínios. Conclusão: A significativa prevalência de sinais e sintomas de ansiedade e depressão nos pacientes em seguimento oncológico, repercute com prejuízo da qualidade de vida.

Palavras-chave: Ansiedade, Depressão, Qualidade de vida, Oncologia.

\begin{abstract}
Objective: To estimate the prevalence of signs and symptoms of anxiety and depression and to assess the quality of life of patients undergoing cancer treatment. Methods: The respective study is a cross-sectional study, including 246 patients who are undergoing cancer follow-up. A questionnaire with sociodemographic variables and clinical information was used, plus the instruments: Hospital Scale for Depression and Anxiety (HADS) and World Health Organization Quality of Life (WHOQOL-bref). Results: The prevalence of moderate to severe anxiety was $33.7 \%(95 \% \mathrm{Cl}: 27.8-39.7)$ and $13.4 \%(95 \% \mathrm{Cl}: 9.1-17.7)$ for moderate depression severe. Quality of life had an average score of 68.52 points. Patients, who presented symptoms of anxiety and moderate to severe depression, had a worse assessment of quality of life in all domains. Conclusion: The significant prevalence of signs and symptoms of anxiety and depression in patients undergoing cancer has repercussions with impaired quality of life.
\end{abstract}

Keywords: Anxiety, Depression, Quality of life, Oncology.

\section{RESUMEN}

Objetivo: Estimar la prevalencia de signos y síntomas de ansiedad y depresión y evaluar la calidad de vida de los pacientes en tratamiento oncológico. Métodos: El estudio respectivo es un estudio transversal, que incluyó a 246 pacientes con cáncer. Se utilizó un cuestionario con variables sociodemográficas e información clínica, más los instrumentos: Escala Hospitalaria de Depresión y Ansiedad (HADS) y Calidad de Vida de la Organización Mundial de la Salud (WHOQOL-bref). Resultados: La prevalencia de ansiedad moderada a severa fue 33,7\% (IC 95\%: 27,8 - 39,7) y 13,4\% (IC 95\%: 9,1 - 17,7) para depresión moderada. grave. La calidad de vida tuvo una puntuación media de 68,52 puntos. Los pacientes, que presentaban síntomas de ansiedad y depresión moderada a grave, tenían una peor valoración de la calidad de vida en todos los dominios. Conclusión: La importante prevalencia de signos y síntomas de ansiedad y depresión en pacientes con cáncer tiene repercusiones en el deterioro de la calidad de vida.

Palabras clave: Ansiedade, Depresíon, Calidad de vida, Oncologia.

${ }^{1}$ Centre de consultation psychologique et éducationnelle (CCPE), Montréal, Québec, Canadá.

*E-mail: barbosa da silva.alyne@ccpeweb.ca

2 Universidade Federal de Pernambuco (UFPE), Recife - PE. 


\section{INTRODUÇÃO}

O câncer é uma doença de elevada incidência mundial e de grande impacto para a saúde pública. A Organização Mundial da Saúde (OMS) projeta para 2030 a ocorrência de 27 milhões de casos novos em todo o mundo, com cerca de 17 milhões de mortes. No Brasil, a estimativa para o triênio 2020-2022 é o surgimento de 625 mil novos casos de câncer. Atualmente, as neoplasias são a segunda principal causa de morte no país, responsáveis por 190 mil óbitos a cada ano (OLIVEIRA J, et al., 2018).

Muito embora as estatísticas possam apontar um cenário desfavorável, intervenções para diagnóstico precoce e tratamentos mais eficazes têm favorecido uma melhora na sobrevida e como consequência, observa-se um número maior de pessoas na fase de controle (MILLER KD, et al., 2019). Esse período de seguimento oncológico visa detectar precocemente recidivas, metástases, novo câncer primário, efeitos iatrogênicos, bem como atuar em reabilitação e suporte, oferecer apoio e avaliar as intervenções terapêuticas (QUEIROZ M, 2019). Neste contexto, os pacientes passam periodicamente por exames clínicos e de imagens e pela expectativa do resultado e do impacto deste na sua vida.

Dessa forma, os avanços tecnológicos para diagnóstico do câncer e o seu respectivo tratamento, permite observar um aumento para em torno de $70 \%$ na sobrevida relativa em cinco anos, demonstrando assim uma crescente necessidade de desenvolver meios de melhorar o bem-estar desses indivíduos. Sendo o câncer uma doença sistêmica, a base de tratamento muitas vezes inclui recursos que podem afetar a qualidade de vida e a funcionalidade desses pacientes (FRENSHAM LJ, et al., 2018).

A fase de diagnóstico se caracteriza por uma fase de pensamentos intrusivos sobre o futuro, envolvendo sentimentos de ansiedade, de depressão e de irritabilidade (ANTUNES A, et al., 2016). Apesar dos avanços na sua detecção e controle, o câncer ainda é uma doença que remete à morte, podendo causar sofrimento $e$ trazer desesperança para o paciente e a sua família e aumentar consideravelmente a ansiedade e depressão. Nesse contexto, a morte passa a ser esperada pelo paciente diante de uma doença que coloca sua vida em risco. O medo não termina após uma eventual cura, isso porque sempre existe a possibilidade de uma recidiva (COELHO JCC, et al., 2019).

O homem contemporâneo, representa a morte como um acontecimento pavoroso e medonho, tendendo a não encarar abertamente sua finitude; só eventualmente e com um certo temor é que lançará um olhar sobre a possibilidade de sua própria morte. A consciência de que sua vida está ameaçada por uma doença pode se apresentar como uma dessas eventuais ocasiões. O medo da morte pode englobar o medo de separar-se dos entes próximos amados, o medo da solidão e da interrupção dos projetos e desejos. A cultura, bem como as crenças familiares ou pessoais, influencia as concepções individuas sobre a representação da morte, atribuindo-se personificação, qualidades e formas (BUSA ALA, et al., 2019).

Sinais e sintomas de ansiedade e depressão podem surgir, ocasionando sofrimento físico, psíquico, social e espiritual e acarretando prejuízo funcional, baixa adesão aos tratamentos propostos, redução do autocuidado e diminuição da qualidade de vida, favorecendo um pior prognóstico e contribuindo para maiores morbidades e mortalidade (MONTAZERI A, 2008; LIN M-F, et al., 2011). Quando a ansiedade e o medo se tornam patológicos causam prejuízos na qualidade de vida do paciente, no bem-estar e até mesmo no aproveitamento do seu cotidiano e nas suas atividades de vida diária.

Associado a isto, a presença de doenças crônicas aumenta o risco para depressão e o impacto do câncer na saúde mental tem sido tema de várias pesquisas. Ansiedade e depressão têm forte e independente associação com problemas de saúde mental e carga somática de sintomas. Um número clinicamente relevante de pacientes apresenta ou desenvolve sintomas de ansiedade e depressão com diminuição da qualidade de vida. Logo, a identificação de sintomas de ansiedade e depressão é importante (THALENLINDSTROM A, et al., 2017).

As taxas de prevalência de sofrimento psíquico em pacientes com câncer variam de 22 a $58 \%$ (CARDOSO G, et al., 2016). Estima-se também que, aproximadamente, 20 a $48 \%$ dos pacientes oncológicos preencham os critérios clínicos para ansiedade e/ou depressão (BERGEROT CD, et al., 2014). Porém, a maioria desses estudos é realizada durante a fase de tratamento, sendo escassos os trabalhos que investigam a prevalência de transtornos mentais nos pacientes em seguimento oncológico. 
Em meio aos principais tratamentos clínicos no combate ao câncer encontra-se a quimioterapia que pode ser curativa, adjuvante, neoadjuvante e paliativa. Outros recursos de tratamento muito utilizados no adoecimento oncológico são: a radioterapia, que consiste na utilização de feixes ionizantes de radiação para destruir um tumor ou impedir o crescimento celular maligno; a hormonioterapia um tratamento com a função de inibir atividades dos hormônios que tenham alguma influência no crescimento de um tumor; a terapia alvo que usa de medicamentos compostos de substâncias desenvolvidas para identificar e atacar células cancerígenas; a imunoterapia que visa potencializar o sistema imunológico permitindo combater infecções e outras doenças como câncer e a cirurgia, que pode ser curativa quando a doença é diagnosticada no estágio inicial (KALIKS RA, et al., 2017).

Em decorrência do tratamento e do próprio adoecimento estes indivíduos podem ser acometidos por efeitos adversos que incluem dor, fadiga, possível comprometimento funcional, morbidade, e problemas psicológicos, o que afeta negativamente a qualidade de vida. Segundo a Organização Mundial de Saúde a qualidade de vida é a percepção que o indivíduo tem de sua posição na vida, no contexto de sua cultura, dos sistemas de valores nos quais ele vive, em relação aos seus objetivos, expectativas, padrões e preocupações, envolvendo o bem estar físico, mental assim como seus relacionamentos com a sociedade e com o meio em que vive, o que inclui suas condições de habitação (MAKLUF ASD, et al., 2006).

Considerando que a qualidade de vida dessa crescente parcela da população pode ser prejudicada e que o não reconhecimento de sintomas de transtornos mentais impossibilita seu correto diagnóstico e o encaminhamento para tratamento especializado, são necessárias mais pesquisas que abordem essa temática. Assim, este estudo foi motivado pela magnitude do número de pessoas acometidas por câncer no Brasil e teve como objetivo estimar a prevalência de sinais e sintomas de ansiedade e depressão e avaliar a qualidade de vida de pacientes em seguimento oncológico.

\section{MÉTODOS}

Trata-se de um estudo de corte transversal, estruturado a partir de uma amostra não probabilística, constituída por pacientes em seguimento oncológico, na faixa etária acima de 18 anos. A coleta de dados foi realizada em duas unidades de saúde: um hospital público de referência na região Norte/Nordeste em oncologia e uma clínica especializada em onco-hematologia, ligada a um hospital particular em Recife/Pernambuco.

Para calcular o tamanho amostral, utilizou-se o programa de domínio público Openepi, versão 3.03. O cálculo baseou-se numa prevalência de transtorno afetivo de $20 \%$ em pacientes oncológicos (BERGEROT $C D, 2014)$. Considerando-se um erro alfa de 0,05 e um erro beta de 0,15 (IC=95\%), encontrou-se uma amostra de 246 participantes.

Incluíram-se no estudo pacientes de ambos os sexos, na faixa etária anteriormente citada. O critério de exclusão foi qualquer comprometimento na capacidade de compreensão que impossibilite o entendimento do Termo de Consentimento Livre e Esclarecido (TCLE) e de itens do questionário.

A coleta de dados foi realizada pela pesquisadora principal. A fim de garantir o sigilo e a confidencialidade, a aplicação dos questionários ocorreu individualmente e em local apropriado. Para a coleta, utilizou-se um questionário com perguntas estruturadas, incluindo aspectos sociodemográficos e características clínicas. As variáveis analisadas foram: idade, sexo, cor da pele, local de residência, escolaridade (em anos de estudo), estado civil, filhos, religião e renda. O questionário foi acrescido de dois instrumentos, a Escala Hospitalar de Depressão e Ansiedade (HADS) elaborada para identificar possíveis casos de transtornos de ansiedade e/ou depressão e validada para o Brasil, e o World Health Organization Quality of Life Scale (WHOQOL-bref), desenvolvido pela OMS e validado no Brasil, recomendado para avaliar a qualidade de vida em quatro domínios: físico, psicológico, relações sociais e meio ambiente (ZIGMOND S, SNAITH RP, 1983; THE WHOQOL GROUP, 1998).

A HADS é uma escala de auto-administração e demora cerca de 10 minutos a ser preenchida. Ela é formada por 14 itens com sete questões para ansiedade e sete para depressão, formando duas subescalas que podem ser pontuados de 0 a 3 atingindo no máximo 21 pontos em cada subescala: HADS-A (ansiedade) e HADS-D (depressão). Para cada questão o paciente deve responder sobre a presença ou a frequência dos 
sintomas, existindo quatro possibilidades de resposta. Para cada questão a pessoa deve escolher a resposta que se adapta à forma como se tem sentido durante a última semana. A literatura aponta que o escore igual ou maior que 8 é indicativo de ansiedade moderada a severa e escore igual ou superior a 9 é indicativo de depressão moderada a severa.

O WHOQOL-bref, é uma versão abreviada do WHOQOL elaborada com intuito de tornar mais prática a aplicação deste instrumento de avaliação da qualidade de vida, sem, portanto, comprometer a qualidade dos resultados. Por isso, foi utilizado para a coleta de dados nesta pesquisa. Ele consiste, por sua vez, em um questionário de auto-administração compreendendo 26 perguntas, 2 gerais (uma sobre a percepção da qualidade de vida e outra sobre satisfação com a saúde), e 24 distribuídas em quatro domínios, os quais são: o domínio físico, o psicológico,o das relações sociais e o do meio-ambiente.

Estes domínios são representados por facetas e suas questões formuladas para uma escala de respostas do tipo Likert, com escala de intensidade (nada-extremamente), capacidade (nada-completamente), freqüência (nunca-sempre) e avaliação (muito insatisfeito-muito satisfeito; muito ruim-muito bom). Posteriormente, são calculados os escores de avaliação de cada um dos quatro domínios, acima citados, que tem como valor mínimo zero e o valor máximo 100, em uma escala positiva, ou seja, quanto mais alto o escore, melhor a qualidade de vida naquele domínio.

Todos os pacientes que concordaram em participar da pesquisa assinaram o Termo de Consentimento Livre Esclarecido - TCLE. O estudo atendeu a todos os critérios éticos e teve anuência das duas unidades de saúde e aprovação pelo Comitê de Ética em Pesquisa do Hospital de Câncer de Pernambuco sob o №. 39772214.0.0000.5205.

Foram apresentadas as estatísticas descritivas por meio de distribuição de frequências para as variáveis categóricas e para as médias com suas respectivas variações para as variáveis quantitativas. Na análise da associação de ansiedade e depressão em relação às características biológicas, socioeconômicas e demográficas foram testadas com a aplicação do teste Qui-Quadrado de Pearson. Quanto à associação da depressão e ansiedade e o escore de qualidade de vida, foi utilizado o teste T de Student.

A descrição do escore de qualidade de vida WHOQOL-bref foi representada pela média e desvio padrão por apresentar distribuição normal em todos os domínios. O teste de normalidade aplicado foi o teste de Shapiro-Wilks. Foi aplicado uma análise de regressão logística para o ajuste da associação do escore de qualidade de vida e sintomas de ansiedade e depressão, a fim de controlar possíveis fatores de confusão. A significância estatística adotada no estudo foi de $5 \%(p<0,05)$. O software utilizado na análise foi o STATA versão 12.0.

\section{RESULTADOS}

As prevalências encontradas neste estudo foram de 33,7\% (IC 95\%: 27,8 - 39,7) para ansiedade moderada a severa e 13,4\% (IC 95\%: 9,1 - 17,7) para depressão moderada a severa (Tabela 1). A qualidade de vida teve escore médio de 68,52 pontos.

Tabela 1 - Prevalência de sinais e sintomas de ansiedade e depressão em pacientes pós-tratamento oncológico. Recife, Pernambuco, Brazil, 2015.

\begin{tabular}{lccc}
\hline Sinais e sintomas & Número & $\%$ & IC 95\% \\
\hline Ansiedade & & & \\
\hline Leve & 163 & 66,3 & $60,3-72,2$ \\
Moderada a severa & 83 & 33,7 & $27,8-39,7$ \\
\hline Depressão & & & $82,3-90,9$ \\
\hline Leve & 213 & 86,6 & $9,1-17,7$ \\
\hline Moderada a severa & 33 & 13,4 & \\
\hline
\end{tabular}

Notas: Ansiedade: leve (0-7) / moderada a severa (8-21); Depressão: leve (0-8) / moderada a severa (9-21). Fonte: Silva $A B$, et al., 2021.

Quanto aos aspectos sociodemográficos, os pacientes, em sua maioria, eram idosos $(52,4 \%)$ e do sexo feminino (74\%). Declararam-se brancos $(55,7 \%)$, residentes na Região metropolitana do Recife $(68,7 \%)$, afirmaram ter dez ou mais anos de estudo $(61 \%)$, casados $(59 \%)$, com filhos $(80 \%)$, católicos $(59 \%)$ e com renda de até dois salários mínimos (46\%) (Tabela 2). 
Tabela 2 - Características biológicas, socioeconômicas e demográficas dos pacientes em segmento póstratamento oncológico. Recife, Pernambuco, Brazil, 2015.

\begin{tabular}{|c|c|c|}
\hline Características & Número & $\%$ \\
\hline \multicolumn{3}{|l|}{ Faixa etária } \\
\hline 18 a 39 anos & 16 & 6,5 \\
\hline 40 a 59 anos & 101 & 41,1 \\
\hline 60 anos ou mais & 129 & 52,4 \\
\hline \multicolumn{3}{|l|}{ Sexo } \\
\hline Feminino & 182 & 74,0 \\
\hline Masculino & 64 & 26,0 \\
\hline \multicolumn{3}{|l|}{ Cor da pele } \\
\hline Branca & 137 & 55,7 \\
\hline Parda ou negra & 68 & 27,6 \\
\hline Outra & 41 & 16,7 \\
\hline \multicolumn{3}{|l|}{ Local de residência } \\
\hline $\mathrm{RMR}^{1}$ & 169 & 68,7 \\
\hline Interior na zona urbana & 61 & 24,8 \\
\hline Interior na zona rural & 16 & 6,5 \\
\hline \multicolumn{3}{|c|}{ Escolaridade (anos de estudos) } \\
\hline Analfabeto & 18 & 7,3 \\
\hline De 1 a 4 anos & 44 & 17,9 \\
\hline De 5 a 9 anos & 34 & 13,8 \\
\hline De 10 a 12 anos & 77 & 31,3 \\
\hline 12 anos ou mais & 73 & 29,7 \\
\hline \multicolumn{3}{|l|}{ Estado civil } \\
\hline Solteiro & 52 & 21,1 \\
\hline Casado & 145 & 59,0 \\
\hline Separado/Viúvo & 49 & 19,9 \\
\hline \multicolumn{3}{|l|}{ Filhos } \\
\hline Sim & 196 & 79,7 \\
\hline Não & 50 & 20,3 \\
\hline \multicolumn{3}{|l|}{ Religião } \\
\hline Não tem & 6 & 2,4 \\
\hline Católica & 145 & 59,0 \\
\hline Evangélica & 59 & 24,0 \\
\hline Outras & 36 & 14,6 \\
\hline \multicolumn{3}{|l|}{ Renda } \\
\hline Até $2 \mathrm{SM}^{2}$ & 114 & 46,3 \\
\hline De 2 a menos de 5 SM & 55 & 22,4 \\
\hline De 5 a menos de $10 \mathrm{SM}$ & 37 & 15,0 \\
\hline $10 \mathrm{SM}$ ou mais & 40 & 16,3 \\
\hline
\end{tabular}

Legenda: ${ }^{1}$ RMR- Região Metropolitana do Recife; ${ }^{2 S M-~ S a l a ́ r i o ~ m i ́ n i m o ~ v i g e n t e . ~ F o n t e: ~ S i l v a ~ A B, ~ e t ~ a l ., ~} 2021$.

Segundo as características clínicas, foram prevalentes os cânceres de mama (51,6\%), gastrointestinal $(12,2 \%)$ e próstata (11,4\%). Quanto ao período de conclusão do tratamento: menos de dois anos (41,1\%), de 2 a 4 anos (36,2\%) e acima de 5 anos $(22,7 \%)$ dos pacientes. Houve igual distribuição entre o serviço público e a clínica particular.

$\mathrm{Na}$ análise de qualidade de vida, o instrumento WHOQOL-bref foi dividido segundo os domínios: Qualidade de vida geral, físico, psicológico, relações sociais e meio ambiente. Considerando que o escore de qualidade de vida é medida por uma escala de 0 a 100, observa-se que a pior avaliação entre os domínios foi o físico, com uma média de 64 pontos, aproximadamente, e a melhor avaliação foi em relação ao domínio do meio ambiente. No geral a qualidade de vida teve escore médio de 68,52 pontos (Tabela 3).

Tabela 3 - Descritiva do escore de qualidade de vida WHOQOL-bref dos pacientes em segmento póstratamento oncológico segundo os domínios. Recife, Pernambuco, Brazil, 2015.

\begin{tabular}{l}
\hline Domínios \\
\hline Qualidade de vida geral \\
Físico \\
Psicológico \\
Relações sociais \\
Meio ambiente
\end{tabular}

Média \pm dp
$68,52 \pm 7,15$
$63,78 \pm 7,90$
$68,15 \pm 8,50$
$69,33 \pm 10,28$
$74,23 \pm 12,86$

Notas: Escore de qualidade de vida medida em uma escala de 0 a 100; dp: desvio padrão.

Fonte: Silva AB, et al., 2021. 
$\mathrm{Na}$ associação entre depressão e ansiedade com os aspectos sociodemográficos e as características clínicas, observou-se diferença estatisticamente significante quando comparadas as prevalências de ansiedade nos níveis moderados a severa, em relação ao sexo e estado civil: as mulheres e as pessoas que se declararam solteiras apresentaram maior ansiedade. Quanto à renda, a significância estatística foi limítrofe, quanto maior a renda menor a frequência de ansiedade moderada a severa (Tabela 4).

Em relação à depressão, os resultados foram semelhantes ao encontrado na análise da ansiedade, com diferença estatisticamente significante quando comparada a frequência de depressão moderada a grave em relação ao sexo e significância limítrofe em relação ao estado civil. A ocorrência de depressão moderada a grave foi mais frequente entre as mulheres e os solteiros (Tabela 4).

Tabela 4 - Associação entre depressão e ansiedade com as características biológicas, socioeconômicas e demográficas dos pacientes em segmento pós-tratamento oncológico. Recife, Pernambuco, Brazil, 2015.

\begin{tabular}{|c|c|c|c|c|}
\hline \multirow[b]{2}{*}{ Características } & \multicolumn{2}{|c|}{ Ansiedade } & \multicolumn{2}{|c|}{ Depressão } \\
\hline & $\begin{array}{c}\text { Moderada a severa } \\
\text { Número (\%) }\end{array}$ & p-valor & $\begin{array}{c}\text { Moderada a severa } \\
\text { Número (\%) }\end{array}$ & p-valor \\
\hline \multicolumn{5}{|l|}{ Faixa etária } \\
\hline 18 a 59 anos & $46(39,3 \%)$ & 0,078 & $20(17,1 \%)$ & 0,107 \\
\hline 60 anos ou mais & $37(28,7 \%)$ & & $13(10,1 \%)$ & \\
\hline \multicolumn{5}{|l|}{ Sexo } \\
\hline Feminino & $72(39,6 \%)$ & $0,001^{*}$ & $32(17,6 \%)$ & $0,001^{*}$ \\
\hline Masculino & $11(17,2 \%)$ & & $1(1,6 \%)$ & \\
\hline \multicolumn{5}{|l|}{ Cor da pele } \\
\hline Branca & $45(32,8 \%)$ & 0,499 & $17(12,4 \%)$ & 0,874 \\
\hline Não branca & $21(30,9 \%)$ & & $10(14,7 \%)$ & \\
\hline Outra & $17(41,5 \%)$ & & $6(14,6 \%)$ & \\
\hline \multicolumn{5}{|l|}{ Local de residência } \\
\hline RMR & $54(32,0 \%)$ & 0,380 & $19(11,2 \%)$ & 0,139 \\
\hline Interior & $29(37,7 \%)$ & & $14(18,2 \%)$ & \\
\hline \multicolumn{5}{|l|}{ Escolaridade } \\
\hline Menos de 4 anos & $26(41,9 \%)$ & 0,176 & $11(17,7 \%)$ & 0,512 \\
\hline De 5 a 9 anos & $13(38,2 \%)$ & & $4(11,8 \%)$ & \\
\hline 10 anos ou mais & $44(29,3 \%)$ & & $18(12,0 \%)$ & \\
\hline \multicolumn{5}{|l|}{ Estado civil } \\
\hline Solteiro & $25(48,1 \%)$ & $0,034^{*}$ & $12(23,1 \%)$ & 0,067 \\
\hline Casado & $41(28,3 \%)$ & & $15(10,3 \%)$ & \\
\hline Separado/Viúvo & $17(34,7 \%)$ & & $6(12,2 \%)$ & \\
\hline \multicolumn{5}{|l|}{ Filhos } \\
\hline Sim & $67(34,2 \%)$ & 0,771 & $28(14,3 \%)$ & 0,427 \\
\hline Não & $16(32,0 \%)$ & & $5(10,0 \%)$ & \\
\hline \multicolumn{5}{|l|}{ Religião } \\
\hline Não tem & $2(33,3 \%)$ & 0,731 & $2(33,3 \%)$ & 0,204 \\
\hline Católica & $45(31,0 \%)$ & & $17(11,7 \%)$ & \\
\hline Evangélica & $23(39,0 \%)$ & & $11(18,6 \%)$ & \\
\hline Outras & $13(36,1 \%)$ & & $3(8,3 \%)$ & \\
\hline \multicolumn{5}{|l|}{ Renda } \\
\hline Até 2 SM & $44(38,6 \%)$ & 0,068 & $20(17,5 \%)$ & 0,201 \\
\hline De 2 a menos de 5 SM & $21(38,2 \%)$ & & $6(10,9 \%)$ & \\
\hline $5 \mathrm{SM}$ ou mais & $18(23,4 \%)$ & & $7(9,1 \%)$ & \\
\hline
\end{tabular}

Legenda: Associação estatisticamente significante $(p<0,05)$. Fonte: Silva AB, et al., 2021.

$\mathrm{Na}$ análise da associação entre o escore de qualidade de vida e sinais e sintomas de ansiedade e depressão, observa-se que em todos os domínios analisados do questionário WHOQOL-bref a média da escala foi menor entre os pacientes com presença de ansiedade moderada a severa, resultado também encontrado quando comparada aos sintomas de depressão moderada a severa, o que aponta que os pacientes que apresentavam sintomas de ansiedade e depressão moderada a severa tiveram uma pior avaliação da qualidade de vida em todos os domínios (Tabela 5). 
Tabela 5 - Associação entre depressão e ansiedade com o escore de qualidade de vida WHOQOL-bref entre os pacientes em seguimento pós-tratamento oncológico. Recife, Pernambuco, Brazil, 2015.

\begin{tabular}{lcccccc}
\hline \multirow{2}{*}{ Domínios } & \multicolumn{3}{c}{ Ansiedade } & & \multicolumn{2}{c}{ Depressão } \\
\cline { 2 - 7 } & Leve & $\begin{array}{c}\text { Moderada a } \\
\text { severa }\end{array}$ & p-valor & Leve & $\begin{array}{c}\text { Moderada a } \\
\text { severa }\end{array}$ & p-valor $^{\mathbf{b}}$ \\
\hline Geral & $70,4 \pm 6,58$ & $64,9 \pm 6,87$ & $0,000^{*}$ & $69,7 \pm 6,53$ & $61,1 \pm 6,52$ & $0,000^{*}$ \\
Físico & $64,7 \pm 8,06$ & $62,1 \pm 7,30$ & $0,029^{*}$ & $64,4 \pm 7,69$ & $59,7 \pm 8,15$ & $0,004^{*}$ \\
Psicológico & $70,0 \pm 6,98$ & $64,5 \pm 9,96$ & $0,000^{*}$ & $69,4 \pm 7,53$ & $60,3 \pm 10,2$ & $0,000^{*}$ \\
Relações sociais & $76,1 \pm 12,6$ & $70,4 \pm 12,5$ & $0,002^{*}$ & $75,3 \pm 12,5$ & $67,4 \pm 13,5$ & $0,002^{*}$ \\
Meio ambiente & $71,7 \pm 9,54$ & $64,7 \pm 10,1$ & $0,000^{*}$ & $70,9 \pm 9,70$ & $59,5 \pm 8,28$ & $0,000^{*}$ \\
\hline
\end{tabular}

Legenda: Associação estatisticamente significante $(p<0,05)$; ${ }^{a}$ Associação ajustada por sexo, estado civil e renda; b Associação ajustada por sexo e estado civil. Fonte: Silva AB, et al., 2021.

\section{DISCUSSÃO}

Os achados deste estudo identificaram uma elevada prevalência de sintomas de ansiedade na população estudada, mostrando-se semelhante à encontrada em pesquisas realizadas com pacientes durante a fase de tratamento de câncer, em estudo transversal, utilizando aEscala Hospitalar de Depressão e Ansiedade (HADS), realizado com 200 pacientes em tratamento quimioterápico em que 37,5\% dos pacientes apresentaram quadro de ansiedade e $17 \%$ de depressão, o que pode sugerir que o impacto emocional de vivenciar a doença não se encerra com seu tratamento e perdura durante o período de seguimento (BERGEROT CD, 2014). A prevalência de depressão não se mostrou mais elevada que na população geral, mas aliada aos sintomas de ansiedade, contribuíram para o prejuízo na qualidade de vida.

No acompanhamento clínico é possível perceber que os pacientes após o choque inicial do diagnóstico de uma neoplasia maligna, apresentam respostas emocionais tais como: ansiedade, raiva e depressão, ou algum sintoma emocional de estresse. Isto repercute na adesão ao tratamento, duração das internações, qualidade de vida, prognóstico, sobrevida à doença e após o tratamento(SILVA SS, AQUINO TAA, 2008; BULTZ BDJC, 2011). Vivenciar conflitos emocionais desencadeia sentimentos de desamparo, de invasão e exposição física, de perda da autoestima, dos vínculos e desesperança(SILVA NM, et al., 2014).

$\mathrm{Na}$ associação entre o escore de qualidade de vida e sinais e sintomas de ansiedade e depressão, houve prejuízo na qualidade de vida para todos os domínios quando na presença de ansiedade e depressão moderada a severa. Estes dados corroboram com um estudo realizado com 82 pacientes com câncer, utilizando o questionário WHOQOL-bref, o qual encontrou relação significativa entre ansiedade, depressão e qualidade de vida com prejuízo da qualidade de vida quando na presença de sintomas de ansiedade e depressão moderada a severa(BERTAN FC, CASTRO E, 2010). Outros estudos também demonstram que a depressão está frequentemente associada a reduções importantes na qualidade de vida, incluindo uma funcionalidade social prejudicada(RAVINDRAN AV, et al., 2002; CAPELA C, et al., 2009).

A análise dos dados demonstra uma pior avaliação quanto à qualidade de vida para o domínio físico, com uma média de 64 pontos, ao mesmo tempo se verifica que o predomínio foi de $52,4 \%$ de idosos, o que corrobora com a literatura, visto que as neoplasias são mais frequentes em extremos de idade(SOARES MB, et al., 2019). A pior avaliação para o domínio físico pode ser explicada pelos prejuízos físicos advindos com o envelhecimento, o qual tem forte influência nas morbidades, bem como por consequência da toxidade dos tratamentos para o câncer, reduzindo a capacidade de locomoção, aumentando a dependência de terceiros, diminuindo assim a qualidade de vida(JORGE LLR, SILVA SD, 2010).

Dentre os tipos de câncer citados, o de mama apresentou-se em maior frequência, o qual é o mais prevalente entre as mulheres(OLIVEIRA J, et al., 2018). Isto se justifica, por ser também o sexo feminino mais frequente no estudo. As mulheres apresentaram uma maior prevalência de ansiedade e depressão moderada a severa quando comparada aos homens, assim como a frequência de ansiedade moderada a severa foi mais frequente entre os solteiros, entretanto a depressão teve significância limítrofe em relação ao estado 
civil. Estes dados encontram correlação com estudos que mostram que a depressão, acompanhada dos transtornos da ansiedade, acomete principalmente as mulheres e indivíduos solteiros(GAMA MMA, et al., 2008; SANTOS EG, SIQUEIRA MM, 2010).

O estudo teve como limitação o seu próprio desenho visto que foi elaborado para estimar prevalência e não para análise de fatores associados. No entanto, estudos com o desenho mais apropriado para este objetivo são importantes para corroborar para uma melhor compreensão do processo saúde-doença no período de seguimento oncológico, visto que há uma escassez de estudos sobre os aspectos emocionais que perpassam a fase de seguimento oncológico.

\section{CONCLUSÃO}

O diagnóstico de câncer traz repercussões psicológicas e emocionais para população, as quais podem ser explicadas pelo grande receio em adoecer, ficar desamparado, sofrer impactos em sua imagem corporal e, por fim, pelo medo de morrer. A significativa prevalência de sinais e sintomas de ansiedade e depressão nos pacientes em seguimento oncológico repercute com prejuízo na qualidade de vida, ou seja, os impactos advindos dos aspectos emocionais envolvidos na dinâmica do seguimento oncológico são importantes para o cenário de saúde desta fase do acompanhamento, para que se busque minimizar os fatores de risco em saúde mental e se possibilite ao paciente uma sobrevida com mais qualidade.

\section{REFERÊNCIAS}

1. ANTUNES A, REMONDES COSTAS. Sofrimento com a doença e sintomatologia psicopatológica em doentes oncológicos: prevalência, relação e diferenciação. Rev Psiquiatr Cons Ligaçắo. 2016.

2. BERGEROT CD, et al. Assessment of anxiety and depression in cancer patients: A psychometric comparison. Psico-USF. 2014;19.

3. BERTAN FC, CASTRO E. Quality of life, anxiety and depressions indicators and sexual satisfaction in adult patients with cancer. Salud Soc 2010. 2010;1(2).

4. BULTZ BD, JOHANSEN C. Screening for distress, the 6th vital sign: where are we, and where are we going? Wiley Online Libr. 2011;20 (6):569-571.

5. BUSA ALA, et al. O luto do iovem adulto decorrente da morte dos pais pelo câncer. Rev Psicol: Ciênc e Prof, 2019 ; $39: 1-13$.

6. CAPELA C, et al. Association of quality of life with pain, anxiety and depression. Fisioter e Pesqui. 2009;16(3):263-268.

7. CARDOSO $\mathrm{G}$, et al. Depression and anxiety symptoms following cancer diagnosis: a cross-sectional study. Psychol Health Med. 2016; 21(5):562-570.

8. COELHO JCC, et al. Sintomas de ansiedade e depressao em pacientes oncológicos atendidos por equipe de psicologia.Rev InterCiencia-IMES Catanduva, 2019;1(2): 45-45.

9. FRENSHAM LJ, et al. Effect of a 12-Week Online Walking Intervention on Health and Quality of Life in Cancer Survivors: A Quasi-Randomized Controlled Trial, Switzerland. International Journal of Environmental Research and Public Health, 2018; 15: 1-17.

10. GAMA MMA, et al. Trait anxiety in brazilian university students from Aracaju. Rev Psiquiatr do Rio Gd do Sul. 2008;30(1):1924.

11. JORGE LLR, SILVA SD. Evaluation of the Quality of Life of Gynecological Cancer Patients Submitted to Antineoplastic Chemotherapy. Rev Latino-Am Enferm. 2010;18(5):849-855.

12. KALIKS RA, et al. Diferenças no tratamento sistêmico do câncer no Brasil: meu SUS é diferente do teu SUS, São Paulo. Revista Braz J Oncol, 2017; 13: 1-12

13. LIN M-F, et al. A randomised controlled trial of the effect of music therapy and verbal relaxation on chemotherapy-induced anxiety. J Clin Nurs. 2011;20(8):988-999.

14. MAKLUF ASD, et al. Avaliação da qualidade de vida em mulheres com câncer de mama, Minas Gerais. Revista Brasileira de Cancerologia, 2006; 52: 49-58.

15. MILLER KD, et al. Cancer treatment and survivorship statistics, 2019. CA: a cancer journal for clinicians. 2019 Sep $1 ; 69(5): 363-85$.

16. MONTAZERI A. Health-related quality of life in breast cancer patients: A bibliographic review of the literature from 1974 to 2007. J Exp Clin Cancer Res. 2008;27(1).

17. OLIVEIRA J, et al. Search for oncological care: perception of patients and family members. Journal of Nursing UFPE on line. 12(4): 938-946.

18. QUEIROZ M. Post-therapeutic Follow-up of the Oncologic Patient - Shared Care -. Rev Lusófona 2010:199.

19. RAVINDRAN AV, et al. Stress, coping, uplifts, and quality of life in subtypes of depression: A conceptual frame and emerging data. J Affect Disord. 2002;71 (1-3):121-130.

20. SANTOS EG, SIQUEIRA MM. Prevalence of mental disorders in the Brazilian adult population: a systematic review from 1997 to 2009. J Bras Psiquiatr. 2010;59(3):238-246.

21. SILVA NM, et al. Depressão em adultos com câncer. Ciência Atual. 2014 Mar 6:3(1).

22. SILVA SS, AQUINO T.A.A. Patients with cancer: cognitions and emotions coming from diagnosis. Rev Bras Ter Cogn. 2008;2(4).

23. SOARES MB, et al. Efeito do Gengibre (Zingiber officinale) na qualidade de vida do paciente em quimioterapia/Effect of ginger (Zingiber officinale) in patient quality of life in chemotherapy. Brazilian Journal of Development. 2019 Oct 10;5(10):189889002 .

24. THALEN-LINDSTROM A, et al. Development of anxiety, depression and health-related quality of life in oncology patients without initial symptoms according to the hospital anxiety and depression scale: a comparative study. Acta Oncol. 2017;56:1094-1102.

25. THE WHOQOL GROUP. Development of the World Health Organization WHOQOL-BREF quality of life assessment. Psychol Med. 1998;28(3):551-558.

26. ZIGMOND AS, SNAITH RP. The hospital anxiety and depression scale (HADS). Acta Psychiatr Scand. 1983;67(361$370): 361-370$. 